\title{
Green Identity, Myth or Reality: An Abstract
}

\author{
Samreen Ashraf and Maria Musarskaya
}

\begin{abstract}
Green consumerism has been on the rise over the last few decades as a consumer-driven campaign as well as a topic of interest in academic research. Nevertheless, industry experts and researchers alike are yet to create a unifying, consumer-approved definition of what it means to have a green identity. Millennials are a developing consumer base which is not only the most connected but is also the most aware of the green consumption culture. Therefore, this research aims to explore the perceptions of the university students who are classified as millennials in order to identify what it means for them to be a green consumer. Furthermore, this research aims to understand consumers' perceptions of 'green identity', how different or similar these consumers see themselves in comparison to other consumers who are more or less green and how do they identify themselves as green consumers during their university life, by linking self-perception theory and self-identity concept. In the first phase of the study, in-depth interviews are conducted with first and final year undergraduate students to examine how their perception of green identity changes over the span of a university's undergraduate degree. Preliminary results display a list of characteristics that are perceived to be and are part of green consumer identity. Furthermore, preliminary results indicate that family, friends, higher education institutions, existing initiatives, and the media influence the formation of a student's green identity. The second phase of the study will be used to test the proposed conceptual framework using quantitative method.
\end{abstract}

References Available Upon Request

S. Ashraf $(\bowtie) \cdot$ M. Musarskaya

Bournemouth University, Bournemouth, UK

e-mail: sashraf@bournemouth.ac.uk; mmusarskaya@bournemouth.ac.uk 